\title{
Hohe Anforderung an Aufklärung Patient möchte gegen ärztlichen Rat die Klinik verlassen
}

Gericht spricht Patienten hohes Schmerzensgeld und Schadensersatzansprüche zu, weil der Arzt den Patienten, der die Klinik gegen ärztlichen Rat verlassen möchte, nicht mit der gebotenen Klarheit darüber aufgeklärt hat, dass aufgrund einer Ummedikation ein gesteigertes Risiko für den Patienten bestand.

\section{Der Fall}

Der Kläger (Patient) leidet an einer seltenen angeborenen Herzerkrankung (Noncompaction Kardiomyopathie), die eine Herzmuskelschwäche und schwere Herzrhythmusstörungen zur Folge hat. Seit 2004 befand er sich deswegen wiederholt in stationärer und ambulanter Behandlung. Im Frühjahr wurde dem Patienten in einer anderen Klinik ein Defibrillator implantiert. Der Patient nahm den Betablocker Bisoprolol, zeitweise zusammen mit weiteren Medikamenten. Am 01.04.2005 kam es zu einer Herzrhythmusstörung, der Patient verlor das Bewusstsein und stürzte. Mit einer Platzwunde wurde er im Krankenhaus der Beklagten aufgenommen. Von den behandelnden Ärzten wurde der Betablocker Bisoprolol sofort abgesetzt und dem Patienten das Antiarrhythmikum Amiodaron verabreicht. Einen Tag später verließ der Kläger gegen Mittag das Krankenhaus, wobei die Einzelheiten des Verlassens zwischen dem Krankenhaus und dem Patienten streitig sind. In den Behandlungsunterlagen hat der Oberarzt A. folgenden Passus vermerkt: „11:30 Visite Entlassung auf eigenen Wunsch mit der dringenden Ermahnung, sich bei Zunahme der Rhythmusstörungen sofort wieder vorzustellen." Am 04.04.2005 traten um 00:11 Uhr vom Kläger bemerkte Herzrhythmusstörungen auf. Zwischen 18:40 Uhr und 19:13 Uhr kam es zu 6 Episoden mit schweren Herzrhythmusstörungen. Die Angehörigen des Patienten riefen deshalb den Notarzt herbei. Als dieser eintraf, war der Patient bei Herz-Kreislaufstillstand bewusstlos. Der Kläger wurde gegen 20:10 Uhr/20:15 Uhr im Krankenhaus der Beklagten aufgenommen, die vom Notarzt begonnenen Reanimationsmaßnahmen wurden von den Ärzten weitere 15 Minuten fortgeführt. Zwar konnten die Herz-Kreislauffunktionen wieder hergestellt werden. Beim Kläger blieb jedoch eine hypoxische Hirnschädigung mit Tetraparese zurück. Der Kläger befindet sich im Wachkoma.

\section{Die Aufklärung}

Der Oberarzt sagte vor dem erstinstanzlichen Gericht als Zeuge aus, dass er dem Patienten den Therapiewechsel erläutert und klargemacht habe, dass er im Hinblick auf die Umstellung 7 Tage stationär verbleiben solle. Der Kläger habe erwidert, dass es ihm gut gehe, er gleich in der Nähe wohne und nicht so lange stationär verbleiben wolle. In dem etwa 20 Minuten dauernden Gespräch habe er, der Zeuge A., den Kläger ermahnt und darüber aufgeklärt, dass der Defibrillator keine Gewähr dafür geben würde, dass es nicht doch zu Herzrhythmusstörungen mit schweren Organschäden und einem Versterben des Patienten kommen könne. Vor dem Berufungsgericht hat der Oberarzt des Weiteren ausgesagt, dass er dem Kläger erklärt habe, dass die Neueinstellung des Medikaments etwa 7-10 Tage brauchen werde. Der Patient habe erwidert, dass er so lange nicht in der Klinik bleiben wolle. Der Oberarzt habe dem Kläger darauf geantwortet, dass die Ärzte ihm nicht sagen könnten, was bei der Umstellung des Medikaments passieren könne. Er habe dem Patienten erklärt, dass sein Defibrillator ihn nicht 100\%ig schützen könne, das Gerät sei keine Garantie. Im Rahmen einer Tachykardie könne er versterben. Er habe dem Kläger auch erklärt, dass es zu bleibenden Organschäden kommen könne. Der Patient habe aber unbedingt nach Hause gewollt. Der Patient verklagte das Krankenhaus auf Schmerzensgeld sowie auf Feststellung der Schadensersatzpflicht für alle weiteren, vergangenen, zukünftigen, materiellen und immateriellen Schäden, welche aus der fehlerhaften Behandlung vom April 2005 resultieren, soweit die Ansprüche nicht auf Sozialversicherungs- träger oder sonstige Dritte übergegangen sind oder übergehen werden.

Das Landgericht beauftragte einen Sachverständigen und vernahm den Oberarzt. Die Klinik wurde zur Zahlung eines Schmerzensgelds von 200000,00 Euro verurteilt und dem Feststellungsantrag bezüglich der weiteren Schäden wurden stattgegeben. Begründet wurde das Urteil damit, dass ein zurechenbarer Behandlungsfehler vorliege. Gegen dieses Urteil legte die Klinik Berufung ein, die jedoch durch das Oberlandesgericht Köln mit Urteil vom 06.05.2012 (Az.: 5 U 28/10) zurückgewiesen wurde.

\section{Die Entscheidung}

Auch nach Auffassung der Richter des OLG lag ein zurechenbarer Behandlungsfehler vor, der zur gesundheitlichen Beeinträchtigung des Patienten, d.h. der Hirnschädigung mit Tetraparese bei Wachkoma, geführt hat.

Zunächst sah das Gericht einen Behandlungsfehler darin, dass die erforderliche stationäre Behandlung ab dem 02.04.2005 unterblieben sei, weil der Patient vor dem Verlassen der Klinik gegen ärztlichen Rat nicht ausreichend aufgeklärt worden sei. Der vom Oberlandesgericht beauftragte Sachverständige kam zu dem Ergebnis, dass es nach der Umstellung der Medikation von dem Betablocker Bisoprolol auf Amiodaron einer stationären Überwachung des Klägers von mindestens einer Woche bedurft hätte. Ausschlaggebend hierfür sei die seltene Grunderkrankung des Patienten gewesen, für deren Behandlung keine Leitlinie vorlag und nur geringe Erfahrungswerte bestanden, die Ohnmacht des Patienten am 01.04.2005, das erneute Auftreten einer Tachykardie am Morgen des 02.04.2005 und vor allem die vollständige Änderung der Medikation mit der Entscheidung, den Betablocker zu pausieren und alleine das Antiarrhythmikum Amiodaron zu verabreichen. Der Sachverständige führte aus, dass Amiodaron Herzrhythmusstörungen entgegen der beabsichtigten Wirkung vermehren und verstärken könne. Von diesem medizinischen Ausgangspunkt, d.h. der Notwendigkeit einer stationären Überwachung des Patienten von einer Woche, ging auch die beklagte Klinik im vorliegenden Verfahren aus. Sowohl der Sachverständige im erstinstanzlichen als auch im zweitinstanzlichen Verfahren hätten übereinstimmend dargelegt, dass ein Patient in der Lage des Klägers, der einen Entlassungswunsch äußerte, darüber aufzuklä- 
ren sei, dass die gesundheitliche Entwicklung zurzeit nicht abgeschätzt werden könne, dass es erneut zu erheblichen Rhythmusstörungen mit Konsequenzen bis hin zum Tod kommen könne, dass die Wirkweise der neu verordneten Medikation ungewiss und es daher unsicher sei, ob die Rhythmusstörungen dadurch weniger oder sogar mehr würden. Diesen Anforderungen habe die Aufklärung des Oberarztes nicht entsprochen.

\section{Keine ausreichende Aufklärung}

Ausschlaggebend für das Gericht war, dass der Oberarzt auf das mit der Umstellung der Medikation einhergehende und für die Notwendigkeit einer stationären Überwachung mit entscheidende Risiko, dass Amiodaron Herzrhythmusstörungen entgegen der beabsichtigten Wirkung vermehren oder verstärken könne, gerade nicht hingewiesen habe. Der Hinweis des Oberarztes an den Kläger, dass auftretende Herzrhythmusstörungen vom Defibrillator nicht aufgefangen würden und zu einem Versterben führen könnten, entspräche zwar dem allgemeinen stets bestehenden Risiko des Klägers, schlösse aber die besondere, durch ein gesteigertes Risiko des Auftretens von Herzrhythmusstörungen gekennzeichnete Gefahrenlage nach der Ummedikation nicht ein. Auch der Hinweis des Oberarztes, dass die Ärzte dem Patienten nicht sagen könnten, was bei der Umstellung der Medikation passieren könne, sei zwar auf Grundlage der Ausführungen der Sachverständigen für sich genommen richtig, brächte aber das gesteigerte (und damit aufklärungsbedürftige) Risiko des Auftretens von Herzrhythmusstörungen durch die Aufdosierung von Amiodaron ebenfalls nicht in der gebotenen Klarheit zum Ausdruck. Der Patient habe die Erklärung dahingehend verstehen können und dürfen, dass eine Besserung der Situation nicht sicher sei, ohne dass er deshalb die ernsthafte Möglichkeit einer Verschlechterung seiner Gefährdungslage in Betracht hätte ziehen müssen. Gerade der zuletzt genannte Gesichtspunkt sei aber für die Entscheidung zwischen einem Verlassen des Krankenhauses und der empfohlenen Fortführung der stationären Überwachung von wesentlicher Bedeutung. Darüber hinaus habe der Oberarzt die gegenüber dem Patienten erteilten Hinweise in sachlich nicht zutreffender Weise abgeschwächt, indem er (was er vor dem erstinstanzlichen Gericht bekundet habe) dem Kläger sinngemäß erklärt habe, dass, wenn der Kläger nicht so weit weg wohne, das Hindernis, sich wieder vorzustellen, ja nicht so groß sei. Es läge auf der Hand, dass eine geringe Entfernung zwischen Wohnsitz und Krankenhaus dem Patienten bei lebensbedrohlichen Komplikationen vielfach nicht helfen würde, vor allem dann nicht, wenn der Notarzt nicht rechtzeitig alarmiert wird oder eintrifft und deshalb Reanimationsmaßnahmen bei Herz-Kreislaufstillstand über einen gewissen Zeitraum unterbleiben. Das Gericht ging daher davon aus, dass der Patient bei korrekter Aufklärung im Krankenhaus zur stationären Überwachung geblieben wäre. Es gäbe keine Anhaltspunkte dafür, dass der Kläger das Krankenhaus auch im Falle eines Hinweises auf das gesteigerte Risiko des Auftretens von Herzrhythmusstörungen nach Ummedikation verlassen hätte. Hierfür spräche auch insbesondere die Angabe der Mutter und Betreuerin des Patienten vor dem OLG, dass der Kläger seine Krankheit immer sehr ernst genommen habe. Das Gericht ging weiter davon aus, dass es bei Auftreten der Herzrhythmusstörungen am 04.04.2005 ab 18:40 Uhr unter stationärer Überwachung nicht zu einer Hirnschädigung des Klägers mit Tetraparese bei Wachkoma gekommen wäre. Hierzu hatten die Sachverständigen ausgeführt, dass es eine ganz große Ausnahme sei, dass eine derartige Situation im Krankenhaus nicht beherrscht werden könne. Dass es zu einem hypoxischen Hirnschaden als Folge einer minutenlangen Unterversorgung mit Sauerstoff komme, sei etwas, was im Krankenhaus praktisch nicht eintreten könne. Hierfür wurde u.a. auf die gebotene Monitorüberwachung verwiesen.

\section{Grober Behandlungsfehler}

Das Oberlandesgericht sah in dem Fehler sogar einen groben Behandlungsfehler. Ein grober Behandlungsfehler setzt neben dem eindeutigen Verstoß gegen bewährte ärztliche Behandlungsregeln oder gesicherte medizinische Erkenntnisse voraus, dass der Arzt einen Fehler begangen hat, der aus objektiver Sicht nicht mehr verständlich erscheint, weil er einem Arzt schlechterdings nicht unterlaufen darf. Für die Beurteilung kommt es auf die Gesamtbetrachtung des Behandlungsgeschehens an. Der Sachverständige des Berufungsverfahrens sah in dem Unterlassen einer stationären Überwachung in Folge unzureichender therapeutischer Aufklärung bei gleichzeitig fehlerhaft unterlassener Abfrage und Umprogrammierung des Defibrillators nach der Tachykardie am Morgen des 02.04.2005 ei- nen groben Behandlungsfehler. Es sei eine regelrecht experimentelle Situation mit vielen Fragezeichen gegeben gewesen. Bei dieser besonderen und gefährlichen Sachlage sei es unverständlich, wenn über die Notwendigkeit und Dringlichkeit einer stationären Überwachung nicht vollständig unterrichtet, insbesondere auf das mit der Gabe von Amiodaron einhergehende gesteigerte Risiko des Auftretens von Herzrhythmusstörungen nicht hingewiesen wird. Nach Auffassung des Sachverständigen wäre die Kombinationstherapie die sicherere Vorgehensweise gewesen und daher durch das Absetzen des Bisoprolols von den handelnden Ärzten noch ein zusätzliches Risiko zu Lasten des Patienten begründet worden.

Ein Mitverschulden des Patienten, weil er sich am 04.04.2005 im Anschluss an die um 00:11 Uhr von ihm bemerkten Herzrhythmusstörungen nicht im Krankenhaus vorgestellt habe, wurde vom Gericht ausgeschlossen. Mit Rücksicht auf den Wissens- und Informationsvorsprung des Arztes käme ein Mitverschulden des Patienten nur dann in Betracht, wenn der Arzt ihn über die Sachlage vollständig und für ihn verständlich unterrichtet habe. Daran fehle es hier.

\section{Fazit}

Auch, wenn dem Fall des Urteils ein Patient mit seltener Erkrankung zugrunde lag, macht es deutlich, dass bei Patienten, die auf eigenen Wunsch oder entgegen ärztlichen Rat das Krankenhaus verlassen wollen, besondere Sorgfalt im Hinblick auf die Aufklärung der Konsequenzen und Risiken gelegt werden muss. Hierbei setzt das Gericht äußerst hohe Maßstäbe an. Es erstaunt allerdings immer wieder, wie wenig Verständnis die Richter in Arzthaftungssachen den (mündigen) Patienten zutrauen. Fakt ist jedoch, dass die Ärzte sich an dieser Rechtsprechung messen lassen müssen und daher der Grundsatz gelten muss, lieber mehr, als zu wenig aufklären. Die Aufklärung sollte zu Beweiszwecken gut dokumentiert werden.

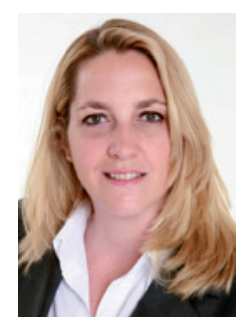

Korrespondenz Dr. iur. Isabel Häser Rechtsanwältin ECOVIS Lüdemann Wildfeuer \& Partner Sonnenstr. 9 80331 München www.ecovis.com 